Disclosure of Interests: None declared

DOI: 10.1136/annrheumdis-2019-eular.2264

\section{THU0230 TNF-ALPHA PROMOTER POLYMORPHISMS (G-308A AND G-238A) ARE ASSOCIATED WITH SUSCEPTIBILITY TO SLE: A HOSPITAL-BASED CASE-CONTROL INVESTIGATION}

MOHAPATRA DEBASISH ${ }^{1}$, Rina Tripathy ${ }^{2}$, Aditya Kumar Panda ${ }^{3}$, Manoj Kumar Parida ${ }^{1}$, Saumya Ranjan Tripathy ${ }^{4}$, Bidyut Kumar Das ${ }^{5} .{ }^{1}$ S.C.B. MEDICAL COLLEGE, Cuttack, Medicine, Cuttack, India; ${ }^{2}$ S.C.B. Medical College, Biochemistry, Cuttack, India; ${ }^{3}$ Khallikote University, Life Sciences, Brahmapur, India; ${ }^{4}$ S.C.B. Medical college, Cuttack, Rheumatology, Cuttack, India; ${ }^{5}$ S.C.B. Medical college, Medicine, Cuttack, India

Background: Tumour necrosis factor- $\alpha(\mathrm{TNF}-\alpha)$ is a proinflammatory cytokine associated with various autoimmune disorders ${ }^{1}$. High levels of TNF- $\alpha$ has been reported in systemic lupus erythematosus (SLE) $)^{2}$. Two functional common polymorphisms (G-238A and G-308A) at promoter region of TNF- $\alpha$ gene have been linked to SLE susceptibility in different populations $s^{3,4}$

Objectives: To investigate association of TNF- $\alpha$ (G-238A and G-308A) polymorphisms with susceptibility/resistance to SLE.

Methods: A total of 102 female SLE patients and 112 age and sex matched healthy controls were enrolled in the study. Patients were examined in detail, physical findings recorded and SLEDAI $2 \mathrm{~K}$ calculated to assess disease severity. TNF- $\alpha$ polymorphisms (G-238A \& G-308A) were typed by polymerase chain reaction and restriction length polymorphism (PCR-RFLP). Plasma level of TNF- $\alpha$ was quantified by ELISA. Statistical analysis was carried out using GRAPH PAD PRISM -7.01.

Results: Mean age of SLE patients and healthy controls was $27.84 \pm 8.83$ and $29.56 \pm 5.48$ years, respectively. At the time of enrolment, mean disease duration of patients was $2.07 \pm 1.13$ years. The mean SLEDAI $2 \mathrm{~K}$ of patients was $16.07 \pm 7.66$. The prevalence of heterozygous mutant and minor allele of TNF- $\alpha$ (G-238A) polymorphisms were significantly higher in SLE patients compared to healthy controls (GA: $P=0.04, \quad O R=2.16$; $A$ : $P=0.02$, OR: 2.09). Furthermore, heterozygous $(G A)$ and minor allele $(A)$ of TNF- $\alpha$ (G-238A) polymorphism were associated with susceptibility to lupus nephritis (GA: $P=0.02, \quad O R=2.89 ; \quad A: P=0.001, \quad O R$ : 2.92). SLE patients displayed higher levels of plasma TNF- $\alpha$ compared to healthy controls. Although the prevalence of heterozygous mutant and minor allele of TNF- $\alpha$ (G-308A) polymorphism was higher in SLE patients, it was not statistically significant. TNF- $\alpha$ (G-238A and G-308A) variants were associated with higher plasma TNF- $\alpha$ in both SLE patients and healthy control. However, no significant association was observed on distribution of TNF$\alpha$ polymorphisms (G-238A and G-308A) with severity of disease activity of SLE patients.

Conclusion: The results of the present study demonstrate that TNF- $\alpha$ (G$238 \mathrm{~A}$ ) variant is associated with higher plasma TNF- $\alpha$ level and increased susceptibility to development of SLE and lupus nephritis.

\section{REFERENCES:}

[1] Aggarwal BB, Gupta SC, Kim JH. Historical perspectives on tumor necrosis factor and its superfamily: 25 years later, a golden journey. Blood. 2012;119(3):651-665.

[2] Studnicka-Benke A, Steiner G, Petera P, Smolen JS. Tumour necrosis factor alpha and its soluble receptors parallel clinical disease and autoimmune activity in systemic lupus erythematosus. Br J Rheumatol. 1996;35 (11):1067-1074.

[3] D’Alfonso S, Colombo G, Della Bella S, Scorza R, Momigliano-Richiardi P. Association between polymorphisms in the TNF region and systemic lupus erythematosus in the Italian population. Tissue Antigens 1996;47(6):551555.

[4] Lee YH, Harley JB, Nath SK. Meta-analysis of TNF-alpha promoter-308 $\mathrm{A} / \mathrm{G}$ polymorphism and SLE susceptibility. Eur J Hum Genet. 2006;14:364-371.

Disclosure of Interests: None declared

DOI: 10.1136/annrheumdis-2019-eular.5559
THU0231

GELSOLIN A NEW BIOMARKER OF DISEASE ACTIVITY IN SLE PATIENTS ASSOCIATED WITH HDL-C

Sandra Parra ${ }^{1,2}$, Mercedes de Las Heras ${ }^{3}$, Pol Herrero ${ }^{4}$, Nuria Amigó ${ }^{5}$, Esperanza Garcés ${ }^{1}$, Josepa Girona ${ }^{3}$, Xavier Correig ${ }^{5}$, Nuria Canela ${ }^{4}$, Antoni Castro1,2. "'Sant Joan" University Hospital, Internal medicine department. Autoimmune diseases unit, Reus, Spain; ${ }^{2}$ Institut Investigacions Sanitàries Pere I Virgili, Reus, Spain; ${ }^{3}$ Universidad Rovira i Virgili, Unitat de Recerca de Lipids $i$ Arteriosclerosis (URLA), Reus, Spain; ${ }^{4}$ Universitat Rovira i Virgili, Centre for Omic Science, Joint Unit, Reus, Spain; ${ }^{5}$ Universitat Rovira i Virgili, Department of Electronic Engineering, Reus, Spain

Background: Recent proteomics techniques have demonstrated that high density lipoprotein (HDL) associated proteins are involved in functions related to systemic inflammatory and immune responses in several pathological conditions, including autoimmune diseases. HDL undergoes structural and functional modifications in systemic lupus erythematous (SLE) patients.

Objectives: To identify potential biomarkers of disease activity analyzing the proteome of HDL particles from SLE patients in clinical remission and when they develop a flare compared to a healthy control group.

Methods: Quantitative proteomic analyses of purified HDL were performed using Tandem Mass Tag (TMT) isobaric tag-labeling and nanoLC-Orbitrap (nLC-MS/MS) from 9 SLE patients in clinical remission when they developed a flare and from 9 healthy controls (9-9-9). We verified the identified proteins by Western blot and ELISA in a cohort of 104 SLE women patients, 46 healthy women and 14 SLE patients when developed a flare.

Results: A total of 83 proteins associated with HDL were identified. We found 17 proteins with a significant fold-change (>1.1) compared with their levels in control patients. In lupus patients experiencing a flare compared with those in remission, we identified 4 proteins with a significant fold-change (C4, Indian Hedgehog protein, S100A8 and gelsolin). Plasma Gelsolin (pGSN) levels were decreased in the 104 SLE patients (176.02 (74.9) $\mathrm{mcg} / \mathrm{l})$ compared with the control group (217.13(86.7) $\mathrm{mcg} / \mathrm{l}) ; \mathrm{p}$ $=0.005$ and when they developed a clinical flare $(104.84(41.7) \mathrm{mcg} / \mathrm{l}) ; \mathrm{p}=$ 0002). pGSN levels were associated with HDL-c levels $(r=0.316, \quad p<$ 0.001). Antimalarial treated patients showed significant higher levels of pGSN (214.56(88.94) $\mathrm{mcg} / \mathrm{l}$ respect $170.35(66.36) \mathrm{mcg} / \mathrm{l}) ; \mathrm{p}=0.017$.

Conclusion: The proteome cargo from HDL differentiates SLE patients from healthy controls. HDL from SLE patients carries proteins that are involved in the activation of the immune system. Decreased pGSN are associated with clinical disease activity in SLE patients. Antimalarial treatment and HDL-c are associated with higher levels of pGSN in SLE patients. pGSN is a potential biomarker of disease activity in SLE patients.

Disclosure of Interests: None declared

DOI: 10.1136/annrheumdis-2019-eular.5958

\section{THU0232 DIFFERENTIAL METHYLATION OF IL8 AND TISSUE FACTOR PROMOTER IN ANTIPHOSPHOLIPID SYNDROME}

Markos Patsouras, Karagianni Panagiota, Paraskevi Kogionou, Panayiotis Vlachoyiannopoulos. National and Kapodistrian University of Athens, Pathophysiology, Athens, Greece

Background: Antiphospholipid syndrome (APS) is an autoimmune thrombophillia characterized by recurrent thromboembolism and/or pregnancy morbidity in the presence of Antiphospholipid antibodies mainly anti-ß2glycoprotein I (anti- $\beta 2 \mathrm{GPI})$, which lead to monocyte and endothelial cell activation and subsequent tissue factor and proinflammatory cytokine expression such us IL-6 and IL-8 (1). Epigenetics describes changes in gene expression without alterations in the genomic sequence. Methylation of DNA at CpG islands by adding a methyl group to the nucleotide cytosine, is one of main epigenetic mechanisms.

Objectives: To explore the possible differential methylation of IL8 and Tissue Factor (F3)gene promoters, which are critical for the pathophysiology of APS.

Methods: Whole blood and serum were isolated from 27 APS patients and 25 healthy donors (HDs). Human umbilical vein endothelial cells (HUVECs) and peripheral blood monocytes were isolated from 3 HDs.

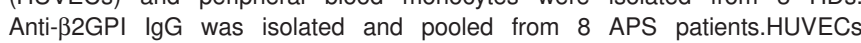
and monocytes were stimulated with a mixture of $\mathrm{IgG}, \beta 2 \mathrm{GPI}$ and CXCL4.Then mRNA was isolated and GPCR was performed for the assessment of IL8 and tissue factor (F3)gene expression.Whole blood DNA from APS patients and HDs,and DNA from the in vitro experiments was isolated and bisulphite treated. The methylation of $1 \mathrm{CpG}$ in the 